\title{
A INTENÇÃO DE USO DE JOGOS VIRTUAIS NAS AULAS DE EDUCAÇÃO FÍSICA ESCOLARES
}

\author{
Pedro da Silva - Universidade FEEVALE - pedrohs_nhrs@ outlook.com \\ Marcelo Curth - Universidade FEEVALE - marcelocurth@ feevale.br \\ Luis Eurico Kerber - Universidade FEEVALE - luislek@ feevale.br \\ Denise Bolzan Berleze - Universidade FEEVALE - deniseberlese@ feevale.br
}

\begin{abstract}
Resumo: O artigo tem como objetivo levantar dados sobre as relações dos construtos facilidade de uso percebida, utilidade percebida e normas subjetivas e sua influência na intenção de uso de jogos virtuais nas aulas de Educação Física por estudantes do curso de Licenciatura em Educação Física de uma universidade do Rio Grande do Sul. Foi realizada uma survey com 141 estudantes do curso. A técnica estatística usada foi modelagem de equações estruturais (MEE) por meio da qual foi testado o modelo conceitual. Os resultados indicam bom ajustamento do modelo de acordo com a literatura. Os construtos referentes a facilidade de uso percebid a e utilid ad e percebida apresentaram efeitos positivos na intenção de uso de jogos virtuais nas aulas de Educação Física, ao contrário das normas subjetivas. Esta pesquisa preenche a lacuna de estudos referentes ao uso de jogos virtuais nas aulas de Educação Física.
\end{abstract}

Palavras-chave: Facilidade de uso percebida. Utilidade Percebida. Intenção de Uso. Tecnologia na Educação.

Abstract: The paper aims to collect data and to identify the influence of perceived ease of use and perceived usefulness concepts and subjective norms while using virtual games in Physical Education classes by undergraduate students attending Physical Education Degree at an university in Rio Grande do Sul. A survey with 141 students from the course was conducted. The conceptual model was tested by the Structural Equation Modeling (SEM) statistical technique. According to the literature, the results found indicate a model's good adjustment. The concepts related to perceived ease of use and perceived usefulness had positive effects on the intention to use virtual games in Physical Education classes, rather than subjective norms. This research fills the gap related to studies of virtual games in Physical Education classes.

Keywords: Perceived ease of use. Perceived Usefulness. Intention to use. Technology on Education.

\section{INTRODUÇÃO}

Os jogos virtuais são atualmente uma tendência tecnológica que visa a diversão e entretenimento, sendo utilizado em inúmeros setores como ferramenta de preparação e capacitação de profissionais. Os jogos podem desenvolver inúmeras valências que estimulam o aprendizado, como concentração, raciocínio rápido, ação e interação, além de serem atrativos por terem características que os tornam divertidos e desafiadores (CANI et al. 2017). Ramos e Segundo (2016) destacam as contribuições dos jogos para o desenvolvimento de habilidades cognitivas, por meio de objetivos que estimulam a tomada de decisão, cumprimento de metas e planejamento de estratégias. Além disso, os jogos são uma ferramenta de aprendizagem coerente na realidade dos estudantes (SENA et al. 2016).

Entretanto, ainda são poucas as propostas da utilização de jogos digitais no âmbito escolar (RAMOS; SEGUNDO, 2016). Essa lacuna é ainda maior na área da Educação Física escolar, onde existe escassez de propostas relacionando jogos digitais e conteúdos contemplados na Educação Física. 
Além disso, diferentes modelos teóricos têm sido desenvolvidos e aplicados para estudar a aceitação e o comportamento de uso de tecnologias em diferentes áreas. Um exemplo é o modelo de aceitação de tecnologia (Technology Acceptance Model, TAM), de Davis (1989), que é considerado um dos mais influentes e mais amplamente utilizados pelos pesquisadores para descrever a aceitação de tecnologia pelos indivíduos. O modelo possibilita uma forte base teórica, além do amplo apoio empírico por meio de validações, aplicações e replicações, pois já foi testado com diferentes amostras e em situações diversas, provando ser válido e confiável (COSTA FILHO et al. 2007).

O modelo será adaptado para esta pesquisa, sendo utilizados os construtos que compõem o modelo: utilidade percebida, facilidade de uso percebid o e a intenção de uso. Além dos construtos do modelo, acreditamos ser importante a inclusão de aspectos relacionados ao local onde os indivíduos estão inseridos, pois acredita-se existir elementos influentes externos na adoção dos jogos, sugerindo a inclusão do construto normas subjetivas no modelo (HA; IM, 2014).

Sendo assim, este artigo buscará analisar a intenção do uso dos jogos digitais por futuros profissionais da área da Educação Física escolar, principalmente pelo fato dos resultados obtidos com jogos digitais em diferentes áreas de ensino serem promissores (AMORIM et al., 2016).

Por fim, os resultados da pesquisa possibilitaram o entendimento sobre a importância da tendência tecnológica no futuro dos cidadãos, considerando ser importante a vivência dessas práticas no currículo de formação daqueles novos profissionais, para que estejam mais preparados para cada evolução que a tecnologia nos propõe.

\section{REFERENCIAL TEÓRICO}

\subsection{Facilidade de Uso Percebida}

A facilidade de uso percebida é o grau em que o usuário acredita que o uso da tecnologia é fácil e livre de esforços e é tida como fator chave no modelo TAM para a aceitação de tecnologias (DAVIS, 1989).

A dificuldade no uso da tecnologia como uma fronteira a ser transposta pelo indivíduo que busca executar as suas tarefas, com determinado esforço e tempo despendido no uso dos sistemas de gestão do conhecimento, tende a inibir mudanças e provocar resistência no processo de adaptação a novos sistemas ou tecnologias (GOODMAN; DARR, 1998).

Segundo Dias (2000), a resistência ao aceitar a utilização de uma nova tecnologia, seguindo a ótica da percepção de sua utilidade ocorre apenas indiretamente, por conta da influência negativa da resistência sobre a facilidade de uso.

Para Aparicio et al. (2017), a facilidade de uso percebid a possui efeito direto sobre a utilidade percebida, sugerindo que quanto maior a facilidade de uso percebida, maior a contribuição no desempenho da tarefa.

Pesquisas com o modelo TAM mostram que, se o sistema for realmente útil, o usuário tende a utilizá-lo, apesar de existirem dificuldades para isso (DIAS, 2000; FERREIRA et al., 2014).

Dessa forma, considerando a importância do construto facilidade de uso percebida, entende-se haver uma relação direta entre a percepção de facilidade de uso e a percepção de utilidade, sugerindo a seguinte hipótese: H1: A facilidade de uso percebida influencia positivamente a percepção de utilidade de tecnologia nas aulas de Educação Física.

\subsection{Intenção de Uso}


A intenção de uso é resultante de um comportamento perante uma determinada ação ou objeto (AJZEN, 1991; DAVIS, 1989; SILVA et al., 2012). Segundo Jarvenpaa et al. (2000), a intenção trata-se do grau em que o indivíduo expressa as suas intenções de utilização de jogos virtuais, send o influenciada pela intenção de comportamento, além de estar diretamente ligada a hábitos, afetos e crenças, definidos como normas subjetivas (AJZEN; FISHBEIN, 1980).

Alguns exemplos de estudos têm pesquisado a intenção de uso de determinada tecnologia, baseando-se nos construtos do TAM. Os estudos foram aplicados em diferentes áreas, como na saúde (ALMEIDA et al., 2013), sistemas de gestão de conhecimento (APARICIO et al., 2017) e em outros ambientes da socied ade (FERREIRA et al., 2014).

Como o construto facilidade de uso percebida é uma das bases sólidas do modelo TAM, acredita-se ser relevante na sugestão da seguinte hipótese: H2: A facilidade de uso percebida influencia positivamente a intenção de uso de tecnologia nas aulas deEducação Física.

\subsection{Percepção de Utilidade}

A percepção de utilidade é definida como o quanto o usuário acredita que ao fazer uso de uma determinada tecnologia ele perceberá melhorias no resultado de atividades. Desse modo, o construto utilidade percebida define-se como uma percepção subjetiva do usuário de que determinada tecnologia possa melhorar seu desempenho em relação ao objeto de uso, geralmente um sistema de informação (COSTA FILHO et al., 2007; KARAHANNA etal., 1999).

Considerando a importância desse construto, pesquisas foram realizadas comparando a sua influência na intenção de uso de novas tecnologias, sendo os casos de Silva et al. (2012), que usaram o TAM para avaliar a utilização do computador na educação por educadores e Hedler et al. (2016), usando o TAM para reconhecer a aceitação de tecnologia de computação em nuvem e transferência de dados em uma escola.

Porém, a escassez de material científico relacionando percepção de utilidade de tecnologias nas aulas de Educação Física sugere a oportunidade do teste da relação, sugerindo a seguinte hipótese: H3: A percepção de utilidade influencia positivamente a intenção de uso de tecnologia nas aulas de Educação Física.

\subsection{Normas Subjetivas}

As normas subjetivas são construtos da Thoery of Reasoned Action (TRA) (AJZEN; FISHBEIN, 1980). A teoria embasou os estudos de Davis (1989) para a elaboração do modelo de aceitação tecnológica, com o objetivo de justificar fatores determinantes para a realização de ações ou de comportamentos dos indivíduos em geral.

Para Karsaklian (2008), as normas subjetivas constituem a base dofuncionamento da sociedade, partindo de um pressuposto em que o impacto de um grupo de influências resulta e corresponde a todos os comportamentos, inclusive no que diz respeito ao comportamento de aceitação ou rejeição de determinada tecnologia.

Dada a sua importância, as normas subjetivas é tema de pesquisas do mundo inteiro, vide Mahon et al. (2006) e Pavlou e Chai (2002), por exemplo, sendo estas pesquisas de ramos diferentes, com comparação de teorias comportamentais, comércio eletrônico de entregas e preferências de comida em pratos prontos ou em pedidos específicos, respectivamente.

No contexto da educação, não foram encontrados estudos que incluíssem as normas subjetivas como um dos construtos a serem testados. Entretanto, Hedler et al. (2016) sugerem que, em estudos futuros sobre aceitação de tecnologias na educação, as normas subjetivas sejam consideradas e envolvidas como fator determinante para a 
aceitação ou rejeição dos sistemas de tecnologia, sugerindo a seguinte hipótese: H4: As normas subjetivas influenciam positivamente a intenção de uso de tecnologia para as aulas de Educação Física.

\section{PROCEDIMENTOS METODOLÓGICOS}

Os participantes da pesquisa são estudantes do curso de Educação Física Licenciatura de uma universidade do Rio Grande do Sul. A amostra contou com 140 estudantes para o levantamento de dados. Segundo Hair Jr. et al. (2009), a amostra deve contar entre 5 a 10 respondentes por parâmetro analisado, sugerindo o alinhamento da amostra coletada e os parâmetros utilizados nesta pesquisa.

A validação do instrumento de pesquisa foi feita por dois profissionais da área da educação em nível de ensino superior, sugerindo ajustes nos enunciados das questões para facilitar sua compreensão e interpretação.

A aplicação do pré-teste ocorreu com 16 estudantes, entre 8 de agosto de 2018 a 4 de outubro de 2018 e teve como objetivo a análise de confiabilidade da escala utilizada, considerando o Alpha de Cronbach. Os resultados apresentaram valores satisfatórios, tend o percepção de utilidade Alpha de 0,7 e 5 indicadores; facilidade de uso contou com Alpha de 0,78 e 3 indicadores; normas subjetivas apresentou o 0,63 e 3 indicadores; e, por fim, intenção de utilização teve Alpha de 0,6 e 3 indicadores.

A etapa para a construção do instrumento de pesquisa contou com a seleção de escalas que foram utilizadas e validad as em outros estudos. As questões foram elaboradas utilizando a escala Likert de 5 pontos, sendo 1 "Discordo totalmente" e 5 "Concordo totalmente".

O instrumento final contou com 16 questões, sendo 13 com os construtos da pesquisa e as últimas três questões sobre o perfil dos respondentes (sexo, a faixa etária e a atuação). Para a coleta foi enviado o link do formulário GoogleForms via WhatsApp e Facebook. Finalizando, com o consentimento do coordenad or do curso, foi enviado o link do formulário para todos os estudantes buscando atingir todos os possíveis resultados da pesquisa.

O curso possui 185 estudantes matriculados e foram respondentes 141 destes, totalizand o um percentual de $76,22 \%$ da amostra.

Para a operacionalização da análise dos dados, foi utilizado o software Microsoft Excel® 2010 e SPSS 20 (Statistical Package for Social Sciences), para os dados descritivos e preparação de dados. O software AMOS тM 20 (Analysis of Moment Structures) foi utilizado para a modelagem de equações estruturais (MEE) por ser o mais adequado para tal tipo de análise (GARSON, 2012). A modelagem de equações estruturais (MEE) pode ser vista, segundo Klem (1995), como extensão da regressão múltipla. Porém, na regressão o pesquisador está interessado em prever uma única variável dependente, enquanto na MEE há mais de uma variável dependente. No caso da regressão múltipla, a variável $\mathrm{X}$ influencia $\mathrm{Y}$. Na MEE, a variável $\mathrm{X}$ influencia $\mathrm{Y}$ e $\mathrm{Y}$ influencia $\mathrm{Z}$.

A MEE não apenas permite o teste confirmatório da estrutura psicométrica de escalas de medida, mas também pode ser utilizada para analisar relações explicativas entre múltiplas variáveis simultaneamente, sejam essas latentes ou observadas.

$\mathrm{O}$ perfil dos participantes apresentou que, dentre seus 141 membros, $78(55,3 \%)$ eram do sexo masculino e na sua maioria na faixa etária entre 20 e 29 anos, somando 101 $(71,6 \%)$ indivíduos. A maioria $(58,2 \%)$ já atuou ou atua na área da Educação Física escolar - 82 indivíduos.

\section{ANÁLISE DE DADOS E RESULTADOS RESULTADOS}

Para a avaliação estatística dos dados coletados, foram utilizados diferentes procedimentos estatísticos de tratamentos dos dados, análise de confiabilidade e validade 
das questões que mediram cada construto utilizado na pesquisa. Ao final, foi utilizada a modelagem de equações estruturais para a realização das análises estatísticas e verificação do modelo conceitual e teste de mediação de variáveis.

Após a análise de outliers, na qual não foram realizadas exclusões de respondentes, foram realizadas as análises de confiabilidade composta (CC) e validade das medidas, sendo realizada pela análise da variância extraída (AVE), como mostra a Tabela 1.

Tabela 1 - Análise dos construtos-Alpha, CC e AVE

\begin{tabular}{c|c|c|c|c|c|c|c|c}
\hline & \multicolumn{4}{|c|}{ Modelo de Medida Inicial } & \multicolumn{3}{c}{ Modelo de Medida Purificado } \\
\cline { 2 - 9 } Constructo & $\begin{array}{c}\mathbf{N}^{\circ} \text { de } \\
\text { itens }\end{array}$ & $\begin{array}{c}\text { Alpha de } \\
\text { Cronbach }\end{array}$ & CC & AVE & $\begin{array}{c}\mathbf{N}^{\mathbf{0}} \text { de } \\
\text { itens }\end{array}$ & $\begin{array}{c}\text { Alpha de } \\
\text { Cronbach }\end{array}$ & CC & AVE \\
\hline PU & 5 & 0,90 & 0,91 & 0,70 & - & - & - & - \\
PFU & 3 & 0,74 & 0,75 & 0,51 & - & - & - & - \\
NS & 3 & 0,79 & 0,80 & 0,57 & - & - & - & - \\
IU & 3 & 0,70 & 0,79 & 0,50 & - & - & - & - \\
\hline Índices sugeridos & & $>\mathbf{0 , 7 0}$ & $>\mathbf{0 , 7 0}$ & $>\mathbf{0 , 5 0}$ & & $>\mathbf{0 , 7 0}$ & $>\mathbf{0 , 7 0}$ & $>\mathbf{0 , 5 0}$ \\
\hline
\end{tabular}

Os indicadores dos construtos apresentaram cargas dentro do sugerido, dessa forma, todas as questões foram mantidas.

Após a purificação dos dados apresentados na Tabela 1, outro teste VD foi aplicado, pois, seguindo a interpretação, os valores de $\mathrm{R}^{2}$ devem ser menores do que os valores de AVE. Para isso, é necessário avaliar a diferença do $\mathrm{x}^{2}$ dos pares de construtos para dois modelos diferentes, sendo sugerida a utilização do teste de Bagozzi e Phillips (1982). Em relação ao modelo, foi avaliado o par: PFU? $\mathrm{IU}, \mathrm{AVE}=0,51$ e $\mathrm{R}^{2}=0,62$ (Tabela 2).

Tabela 2 - Teste de validade discriminante

\begin{tabular}{c|c|c|c|c}
\hline Constructo & PU & PFU & NS & IU \\
\hline PU & $\mathbf{0 , 6 7}$ & & & \\
PFU & 0,46 & $\mathbf{0 , 5 1}$ & & \\
NS & 0,31 & 0,30 & $\mathbf{0 , 5 7}$ & \\
IU & 0,66 & $\mathbf{0 , 6 2}$ & 0,27 & $\mathbf{0 , 5 0}$ \\
\hline \multicolumn{3}{l}{}
\end{tabular}

Para uma nova verificação da validade discriminante dos construtos PFU?IU foi utilizado o teste proposto por Bagozzi e Phillips (1982). Primeiramente, foi testado um modelo livre sem parâmetros fixados para a obtenção do qui-quadrado do modelo livre, sendo o segundo teste com parâmetros fixados em 1. Foi possível verificar a diferença entre o valor fixo em 1 e livre. Também foi identificado um nível de significância $0,13, o$ que justifica que existe validade discriminante e convergente entre os dois construtos, pois não apresentaram valor superior ao corte de 3,84 (BAGOZZI; PHILIPS, 1982).

$\mathrm{Na}$ Tabela 3 são apresentados os coeficientes padronizados e respectivo nível de significância. É possível observar que foram obtidas três hipóteses suportadas e apenas uma não suportada.

Tabela 3 - Cargas dos construtos do modelo conceitual

\begin{tabular}{c|c|c|c|c}
\hline \multicolumn{2}{c|}{ Hipóteses } & \multicolumn{3}{|c}{ Valores } \\
\cline { 3 - 5 } \multicolumn{2}{c|}{} & $\begin{array}{c}\text { Coeficientes } \\
\text { padronizados }\end{array}$ & *Sig & $\begin{array}{c}\text { Suporte para } \\
\text { hipótese }\end{array}$ \\
\hline H1 & PFU - PU & 0,095 & 0,001 & Suportada \\
H2 & PFU - IU & 0,113 & 0,009 & Suportada \\
H3 & PU - IU & 0,122 & 0,001 & Suportada \\
H4 & NS - IU & 0,075 & 0,990 & Não Suportada \\
\hline
\end{tabular}

V. $18 \mathrm{~N}^{\mathrm{o}} 1$, julho, 2020

DOI: 
*Sig = significância.

A hipótese $\mathrm{H} 1$ tratou da relação entre percepção de facilidade de uso e percepção de utilidade. A percepção de facilidade de uso apresentou um efeito direto e positivo na percepção de utilidade, com valores de $\beta=0,09$ e significância $0,001(\mathrm{p}<0,05)$, suportando a hipótese.

Estudos anteriores encontraram influências positivas da percepção de facilidade de uso na utilidade percebida (DAVIS, 1986; PARK, 2010; VENKATESH; DAVIS, 2000; VENKATESH, 1999). Desse modo, se um sistema não requer muito esforço físico nem mental, o utilizad or irá encontrar nele uma utilidade para atingir os seus objetivos ou aumentar o desempenho em alguma tarefa (VILAR, 2013).

A hipótese $\mathrm{H} 2$ tratou da influência da facilidade de uso percebida na intenção de uso. A percepção de facilidade de uso apresentou um efeito direto e positivo na intenção de uso, com valores de $\beta=0,113$ e significância 0,009 ( $p<0,05)$, suportando a hipótese.

A influência da facilidade de uso percebida no contexto do TAM foi validada em pesquisas anteriores (DAVIS, 1986; DAVIS et al., 1989; VENKATESH, 1999; VENKATESH; DAVIS, 2000), bem como em resultados de pesquisas de Vilar (2013) evidenciam a influência desses construtos na área de compras pela internet.

Se um sistema for de demasiada complexidade ou dificuldade de utilização, esperamos que não seja utilizado caso haja um método alternativo. As tecnologias fáceis de usar tendem a ser mais utilizadas do que as mais complexas ou de difícil uso, independentemente da utilid ade percebida (VILAR, 2013).

A hipótese $\mathrm{H} 3$ buscou reconhecer se existe influência positiva da utilidade percebida nas intenções de uso de jogos virtuais na Educação Física escolar. A utilidade percebida apresentou um efeito direto e positivo na intenção de uso, com valores de $\beta=0,122$ e significância $0,001(p<0,05)$, suportando a hipótese.

Outros estudos encontraram uma significante relação entre os construtos de utilidade percebida e sua influência na intenção comportamental de uso, indicando que um aumento de percepção de utilid ade consequentemente aumenta as intenções de uso de tecnologia (CHAU;HU, 2002; DAVIS et al., 1989; HU et al., 1999; MOON; KIM, 2001). Desse modo, podemos evidenciar a influência positiva da utilidade percebida nas intenções de uso de jogos virtuais na Educação Física escolar, visto que, quanto maior a percepção de utilidade sobre determinado jogo, maior a intenção de uso nas aulas de Educação Física.

A hipótese $\mathrm{H} 4$ buscou reconhecer a influência das normas subjetivas nas intenções de uso de jogos virtuais nas aulas de Educação Física. As normas subjetivas não apresentaram um efeito direto e positivo na intenção de uso, com valores de $\beta=0,075$ e significância $0,99(\mathrm{p}<0,05)$, não suportando a hipótese.

A combinação do construto normas subjetivas juntamente do modelo TAM foi revisada e verificada em estudos científicos (GUTMAN; JOIA; MORENO JR., 2014; LEE, 2009; TAYLOR; TODD, 1995; WU; CHEN, 2005). Outros estudos também não obtiveram sucesso ao procurar influência positiva das normas subjetivas nas intenções de uso de determinad as tecnologias (CHAU; HU, 2001; GUTMAN; JOIA; MORENO JR., 2014; KURTZ et al., 2015; TAYLOR; TODD, 1995). Venkatesh e Davis (2000) argumentam que a norma subjetiva pode ser um fator extremamente significante em ambientes em que o uso do sistema é mand atório, o que não configura o caso em análise.

Ademais, os jogos digitais nas aulas de Educação Física estão em processo de difusão, portanto, ainda não existe pressão para que essas tecnologias sejam aceitas nas aulas, o que pode mudar, considerand o os estímulos das escolas para que os objetivos e estratégias da Base Nacional Comum Curricular sejam contemplados nas aulas, onde constam jogos eletrônicos a partir do $6^{\circ}$ ano do Ensino Fundamental. 
Dessa forma, é perfeitamente razoável que o efeito das normas subjetivas na intenção de uso de jogos virtuais nas aulas de Educação Física não seja estatisticamente significante.

Os indicadores de desempenho do modelo conceitual apresentam a diferença por graus de liberdade com significância menor do que 5, o que indica uma boa base para qualidade dos outros índices. O GFI apresentou valor de 0,88 , indicando bom ajuste, pois o valor está próximo de 0,90 . O valor de RMSEA apresentou-se igual a 0,08 , o que indica o ajuste do modelo com a população pesquisada. Os demais índices apresentaram cargas dentro do sugerido pela literatura (HAIR Jr. et al., 2009), reforçando a adequação do modelo.

\section{CONSIDERAÇÕES FINAIS}

O objetivo deste trabalho foi analisar a tecnologia nas aulas de Educação Física, isto é, reconhecer a influência dos construtos facilidade de uso percebida, utilidade percebida e normas subjetivas nas intenções de uso de jogos virtuais nas aulas de Educação Física. Para isso, com base na literatura existente, desenvolvemos um modelo conceitual com hipóteses e, a partir do teste de modelo de equações estruturais, foi possível verificar a aplicabilidade das hipóteses propostas em consonância com os objetivos da pesquisa.

De modo geral, os resultados obtidos estão em linha com a literatura existente, tanto nas hipóteses suportadas quanto na hipótese não suportada. As hipóteses relacionando os construtos facilid ade de uso percebida, utilid ade percebida e intenção de uso foram todas suportadas, pois o modelo de aceitação tecnológica já foi validado e aplicado em diferentes contextos em estudos já citad os nesta pesquisa. Em contrapartida, a hipótese quatro (H4) não foi suportada, porém, também está de acordo com os poucos estudos encontrados que consideraram normas subjetivas como um construto determinante devido à baixa influência de fatores sociais na decisão intrínseca de comportar-se determinadamente perante a utilização de jogos virtuais nas aulas de Educação Física. Outro fator que pode influenciar é a baixa ou quase nula inserção de conteúdos relacionados a jogos virtuais na Educação Física nos diferentes currículos do curso da universidade.

O modelo conceitual proposto neste artigo contribui para o desenvolvimento de pesquisas acadêmicas, pois conseguiu bons índices de ajustamento, apesar de existirem algumas limitações, como a subjetividade na aplicação do construto normas subjetivas no instrumento de pesquisa, por serem extremamente influenciadas por diferentes crenças, costumes, conhecimentos e vivências pessoais. Além desses fatores, o estudo também contribui para o preenchimento de lacunas existentes em pesquisas sobre as intenções de uso de jogos virtuais nas aulas de Educação Física escolar, por estudantes e futuros profissionais da área. Em estudos futuros, podemos buscar avaliar a intenção de uso dos jogos virtuais nas aulas de Educação Física em outras universidades, ou ainda, avaliar a influência de outros construtos nesta intenção de uso.

\section{REFERÊNCIAS}

AJZEN, Icek. The Theory of Planned Behavior. Organizational Behavior and Human Decision Processes, v. 50, n. 2, p. 179-211, December 1991.

AJZEN, I. \& FISHBEIN, M. Understanding attitudes and predicting social behavior. Englewood Cliffs, NJ: Prentice Hall. 1980.

ALMEIDA, Luana Rodrigues de; SILVA, Ana Tereza Medeiros Cavalcanti da; MACHADO, Liliane dos Santos. Jogos para capacitação de profissionais de saúde na 
atenção à violência de gênero. Revista Brasileira de Educação Médica, v. 37, n.1, p.110-119, 2013.

AMORIM, Myrna Cecília Martins dos Santos; OLIVEIRA, Eloiza Silva Gomes; SANTOS, Joel André Ferreira; QUADROS, João Roberto de Toledo. Aprendizagem e Jogos: diálogo com alunos do ensino médio-técnico. Educação \& Realidade, v. 41, n. 1, p. 91-115, 2016.

BAGOZZI, Richard P.; PHILLIPS, Lynn W. Representing and Testing Organizational Theories: A Holistic Construal. Administrative Science Quarterly, v. 27, p. 459-489, 1982.

CANI, Josiane Brunetti et al. Análise de jogos digitais em dispositivos móveis para aprendizagem de línguas estrangeiras. Revista Brasileira de Linguística Aplicada, v. 17, n. 3, p. 455-481, 2017.

CHAU, Patrick Y.K.; HU, Paul Jen-Hwa. Information Technology Acceptance by Individual Professionals: A Model Comparison Approach. Decision Sciences, v. 32, n. 4, p. 699-719, 2001.

COSTA FILHO, Bento Alves da; PIRES, Péricles José; HERNANDEZ, José Mauro da Costa. Modelo Technology Acceptance Model - TAM aplicado aos Automated Teller Machines - ATM's. Innovation \& Management Review, v. 4, n. 1, p. 40-56, 2007.

DAVIS, Fred D. A technology acceptance model for empirically testing new end user information systems: Theory and results. 1986. Doctoral dissertation - MIT Sloan School of Management, Cambridge, MA, 1986.

Perceived usefulness, perceived ease of use, and user acceptance of information technology. MIS Quarterly, v. 13, n. 3, p. 319-340, 1989.

DAVIS, Fred D.; BAGOZZI, Richard P.; WARSHAW, Paul R. User Acceptance of Computer Technology: A Comparison of Two Theoretical Models. Management Science, v. 35, n. 8, p. 982-1003, 1989.

DIAS, D. S. Motivação e resistência ao uso da tecnologia da informação: um estudo entre gerentes. Rev. adm. contemp., Curitiba, v. 4, n. 2, p. 51-66, Agosto, 2000.

FISHBEIN, Martin; AJZEN, Icek. Belief, attitude, intention, and behavior: An introduction to theory and research. Reading, Mass; Don Mills, Ontario: AddisonWesley Pub. Co., 1975.

GARSON, G. David. Structural Equation Modelling. Asheboro, NC: Statistical Associates Publishers, 2012. Disponível em: http://www.statisticalassociates.com/. Acesso em: 13 maio 2018.

GOODMAN, Paul S.; DARR, Eric D. Computer-aided systems and communities: Mechanisms for organizational learning in distributed environments. MIS Quarterly, p. 417-440, 1998.

GUTMAN, Luis Felipe Dantas; JOIA, Luiz Antonio; MORENO JR., Valter Assis. Antecedentes da intenção de uso de sistemas de home broker sob a ótica dos 
investid ores do mercado acionário. Revista de Administração, v. 49, n. 2, p. 353-368, 2014.

HA, Young; IM, Hyunjoo. Determinants of mobile coupon service adoption: assessment of gender difference. International Journal of Retail \& Distribution Management, v. 42, n. 5, p. 441-459, 2014.

HAIR, Jr. J. F. et al. Análise multivariadada dados. Tradução Adonai Schlup Sant'Anna. 6. ed. Porto Alegre: Bookman, 2009.

HEDLER, Helga Cristina et al. Aplicação do Modelo de Aceitação de Tecnologia à Computação em Nuvem. Perspectivas em Gestão \& Conhecimento, v. 6, n. 2, p. 188 207, 2016

HU, Paul J. et al. Examining the technology acceptance model using physician acceptance of telemedicine technology. Journal of Management Information Systems, v. 16, n. 2, p. 91-112, 1999.

JARVENPAA, Sirkka L.; TRACTINSKY, Noam; VITALE, Michael R. Consumer trust in an internet store. Information Technology and Management, v. 1, n. 1\&2, p. 4571, 2000.

KARAHANNA, Elena; STRAUB, Detmar W.; CHERVANY, Norman L. Information technology adoption across time: a cross-sectional comparison of pre-adoption and posadoption beliefs. MIS Quarterly, v. 23, n. 2, p. 183-218, 1999.

KARSAKLIAN, Eliane. Comportamento do consumidor. $2^{\text {a }}$ ed. São Paulo: Atlas, 2008.

KLEM, L. Path analysis. In: GRIMM, L. G.; YARNOLD, P. R. Reading and understanding multivariate statistics. Washington, DC: American Psychological Association, 1995.

KURTZ, Renata et al. Fatores de impacto na atitude e na intenção de uso do m-learning: um teste empírico. REAd - Revista Eletrônica de Administração, 2015, v. 21, n. 1, p.27-56.

MAHON, Denise; COWAN, Cathal; McCARTHY, Mary. The role of attitudes, subjective norm, perceived control and habit in the consumption of ready meals and takeaways in Great Britain. Food Quality and Preference, v. 17, n. 6, p. 474-481, 2006.

MOON, Ji-Won; KIM, Young-Gul. Extending the TAM for a world-wide-web context. Information \& Management, v. 38, p. 217-230, 2001.

PARK, Namkee. Adoption and use of computer-based voice over Internet protocol phone service: Toward an integrated model. Journal of Communication, v. 60, n. 1, p. 40- 72, 2010.

PAVLOU, P. A.; CHAI, L. What drives electronic commerce across cultures? A cross cultural empirical investigation of The Theory of Planned Behavior. Journal of Electronic Commerce Research, v. 3, n. 4, p. 240-253, 2002. 
RAMOS, Daniela Karine; SEGUNDO, Fabio Rafael. Jogos Digitais na Escola: aprimorando a atenção e a flexibilidade cognitiva. Educação \& Realidade, v. 43, n. 2, p. 531-550, 2016.

SENA, Samara de; et al. Aprendizagem baseada em jogos digitais: a contribuição dos jogos epistêmicos na geração de novos conhecimentos. CINTED - UFRGS, Novas Tecnologias na Educação - RENOTE, v. 14 n. 1, julho, 2016.

SILVA, Patrícia; PIMENTEL, Valdenise; SOARES, Juliana. A utilização do computad or na educação: aplicand o o Technology Acceptance Model (TAM).

Biblionline, v. 8, n. esp., p. 263-272, 2012.

TAYLOR, Shirley; TODD, Peter A. Understanding Information Technology Usage: A Test of Competing Models. Information Systems Research, v. 6, n. 2, p. 144-176, 1995.

VENKATESH, Viswanath. Creation of favorable user perceptions: Exploring the role of intrinsic motivation. MIS Quarterly, v. 23, n. 2, p. 239-260, 1999.

VENKATESH, Viswanath; DAVIS Fred D. A Theoretical Extension of the Technology Acceptance Model: Four Longitudinal Field Studies. Management Science, v. 46, n. 2, p. 186-204, 2000.

VILAR, Márcio Alberto da Silva. Modelo de Aceitação da Tecnologia adaptado às compras online. 2013. 135 fl. Dissertação (Mestrado) - Universidade Fernando Pessoa, Porto, 2013. 